\title{
Effectiveness of evidence-based medicine training for undergraduate students at a Chinese Military Medical University: a self-controlled trial
}

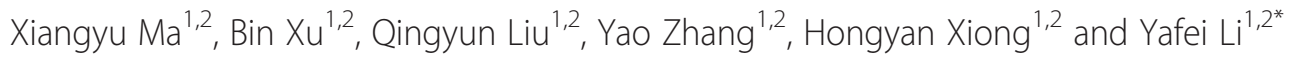

\begin{abstract}
Background: To evaluate the effect of the integration of evidence-based medicine (EBM) into medical curriculum by measuring undergraduate medical students' EBM knowledge, attitudes, personal application, and anticipated future use.

Methods: A self-controlled trial was conducted with 251 undergraduate students at a Chinese Military Medical University, using a validated questionnaire regarding the students' evidence-based practice (EBP) about knowledge (EBP-K), attitude (EBP-A), personal application (EBP-P), and future anticipated use (EBP-F). The educational intervention was a 20-hour EBM course formally included in the university's medical curriculum, combining lectures with small group discussion and student-teacher exchange sessions. Data were analyzed using paired t-tests to test the significance of the difference between a before and after comparison.

Results: The difference between the pre- and post-training scores were statistically significant for EBP-K, EBP-A, EBP-P, and EBP-F. The scores for EBP-P showed the most pronounced percentage change after EBM training (48.97 $\pm 8.6 \%)$, followed by EBP-A (20.83 $\pm 2.1 \%)$, EBP-K (19.21 $\pm 3.2 \%)$, and EBP-F (17.82 $\pm 5.7 \%)$. Stratified analyses by gender, and program subtypes did not result in any significant changes to the results.

Conclusions: The integration of EBM into the medical curriculum improved undergraduate medical students' EBM knowledge, attitudes, personal application, and anticipated future use. A well-designed EBM training course and objective outcome measurements are necessary to ensure the optimum learning opportunity for students.
\end{abstract}

Keywords: Evidence-based medicine, Education, Medical, Evaluation

\section{Background}

Evidence-based medicine (EBM) encourages the assimilation and implementation of the conscientious, explicit, and judicious usage of currently best evidence in making decisions about the care of individual patients [1]. Since EBM was first defined by David Sackett, it is no longer considered acceptable for medical practice to be largely based on clinical anecdotes or expert opinions [2]. Rather, medical decisions should now be based on the best available evidence. EBM is recognized today as a practice that should be learned and applied by all physicians, epidemiologists, and medical administrators worldwide.

\footnotetext{
* Correspondence: liyafei2008@hotmail.com

'Department of Epidemiology, College of Preventive Medicine, Third Military Medical University, Chongqing 400038, People's Republic of China

${ }^{2}$ Center for Clinical Epidemiology and Evidence-based Medicine, Third

Military Medical University, Chongqing, People's Republic of China
}

Furthermore, evidence-based practice (EBP) is also used in low- and middle-income countries [3]. The acquisition of EBM knowledge and skills is also becoming recognized as a core competency that must be acquired by all doctors and medical students. Therefore, an increasing emphasis on training in EBM skills in undergraduate, postgraduate, and continuing medical education programs is becoming more widespread, and implemented, for example via traditional lectures, journal clubs, seminars, and e-learning classes [4-9].

EBM training should be evaluated and guided by evidence of its own effectiveness, including the awareness, attitudes, and competencies regarding EBM of medical students as well as in general practice. Despite the increasing number of medical schools and postgraduate programs that have introduced EBM in their curricula, no studies have evaluated the effectiveness of EBM 
training in China, especially for undergraduate students. Because educational research to support teaching and learning is important and the assessment and evaluation of new approaches is essential for medical education, in this study a self-controlled trial was performed using a validated questionnaire towards the knowledge (EBP-K), attitude (EBP-A), personal application (EBP-P), and future anticipated use (EBP-F) of EBM.

\section{Methods}

\section{Study subjects and design}

The Third Military Medical University, which affiliates to the People's Liberation Army (PLA), is a Chinese military institution of higher learning and trains career physicians for the national military healthcare system. It offers 5- and 8-year programs, in which students receive bachelor and medical doctoral degrees on graduation, respectively. Included in this study were 251 students who had finished the premedical education and clinical courses (Additional file 1). A self-controlled study design was implemented to evaluate the teaching effects. The strategy was approved by the ethics committee of Third Military Medical University (reference number: IRB-TMMU-2012188), and the study was carried out in compliance with the Helsinki Declaration. A written informed consent (Additional file 2) was obtained from all participants which are all adults.

\section{Educational intervention and assessments}

The educational intervention was a 20-hour EBM course formally included in the medical curriculum, including 5 lectures and 2 seminars. First, before the EBM lectures, students were asked to formulate answerable clinical questions based on common clinical scenarios. For example: (1) questions concerning the ability of a test to predict the likelihood of a disease (diagnosis); (2) questions concerning the effectiveness of a treatment or preventative measure (therapy); (3) questions concerning the likelihood of many factors coming to cause an illness (etiology); (4) questions concerning outcome of a patient with a particular condition (prognosis). Second, large group interactive lectures were conducted. The students were trained to develop search strategies according to the PICO format (Patient or Problem, Intervention, Comparison, and Outcome) and to comprehensively retrieve medical databases, including Pubmed, Medline, Embase, Cochrane library, and Web of Science. Teachers then demonstrated how to critically appraise, synthetize, and apply the evidence. Third, small group discussion sessions and student-teacher exchanges were conducted after each lecture. We evaluated the teaching effects of the interventions using validated instruments for the assessment of EBP in terms of knowledge (EBP-K), attitudes (EBP-A), personal application (EBP-P), and anticipated future use (EBP-F), which were employed before and after the EBM training [10]. The assessment questionnaires consisted of 26 questions modified from a reliable assessment tool using a 6point Likert scale (Additional file 3) [11]. The EBP-K section included 5 items (scores: 5-30), the EBP-A and EBP-P sections 6 items each (scores: 6-36), and there were 9 items regarding EBP-F (scores: 9-54). This questionnaire was developed and validated for the assessment of EBM education in the undergraduate learning environment (with similar ages and backgrounds as our student participants) at the University of Hong Kong [11]. The scores in each category were summed for comparisons in the controlled trial.

\section{Statistical analyses}

All statistical analyses were conducted using SAS version 9.2 (SAS Institute, Cary, NC). Mean scores, percentage change, and standard deviations (SD) were calculated to describe the differences for EBP-K, EBP-A, EBP-P, and EBP$\mathrm{F}$ at the pre- and post-stages. In the before and after comparison, paired $t$-tests were used to determine the significance of the difference. All statistical tests were 2-tailed, and $P<0.05$ was interpreted as statistically significant.

\section{Results}

Totally there were 251 undergraduate students participated in this interventional study, completing both the pre- and post-training surveys (response rate $=100 \%$ ). Of the 251 students, 21 are female (8.37\%) and 230 were male (91.63\%). Their ages ranged from 20 to 25 , with a mean of $22(\mathrm{SD}=1)$. Twenty-eight $(11.16 \%)$ students were enrolled in the 8-year program and $223(88.84 \%)$ were in the 5-year program. When investigated, they have the similar schedule of medical courses.

Table 1 presents the comparison of scores for the preand post-training surveys. Statistical significance for the difference between the pre- and post-training scores was detected for all four terms $(\boldsymbol{P}<\mathbf{0 . 0 0 0 1})$. For EBP-K, EBP-P, and EBP-F, the summed post-training scores increased significantly, which means improvements in knowledge, personal application, and anticipated future use. The decreased score for EBP-A represents an improvement in the attitude towards EBM.

As shown in Figure 1, the most pronounced percentage change of scores after an EBM training course was

Table 1 Comparison of scores between survey ahead of the training and survey after the training

\begin{tabular}{llll}
\hline Items & Pre-scores* & Post-scores* & $P$ Value \\
\hline EBP-K & $21.57 \pm 2.99$ & $25.59 \pm 4.80$ & $<0.0001$ \\
EBP-A & $12.91 \pm 5.55$ & $10.08 \pm 6.28$ & $<0.0001$ \\
EBP-P & $14.23 \pm 5.86$ & $20.31 \pm 5.66$ & $<0.0001$ \\
EBP-F & $39.56 \pm 6.47$ & $44.85 \pm 7.97$ & $<0.0001$
\end{tabular}

*pre-scores refer to the scores ahead of the training; post-scores refer to the scores after the training. 


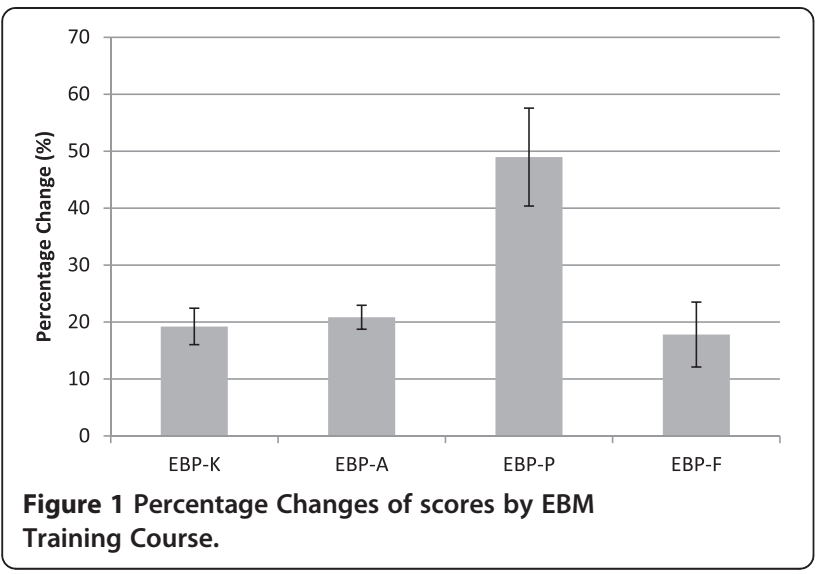

EBP-P $(48.97 \pm 8.6 \%)$, followed by EBP-A $(20.83 \pm 2.1 \%)$, EBP-K $(19.21 \pm 3.2 \%)$, and EBP-F $(17.82 \pm 5.7 \%)$. Furthermore, stratified analyses were conducted. The percentage change of scores, were similar when stratified by gender, and program subtypes (Table 2).

\section{Discussion}

To the best of our knowledge, this study represents the first large interventional trial regarding EBM training conducted in mainland China. The results indicate that significant improvements could be achieved in terms of knowledge, attitudes, personal application skills, and future use in clinical practice through EBM courses for upperclassmen who have completed their premedical education and clinical courses. These results indicate the importance of EBM training for undergraduates, and demonstrate that undergraduate training may serve as an active and useful measure to bridge the gap between medical students and doctors and foster the implementation of EBM.

Considering the high impact of EBP, physicians are increasingly being encouraged to apply EBM. Worldwide, numerous initiatives have emerged to promote EBM including traditional lectures, journal clubs, seminars, workshop, e-learning classes, and competitions [12-18].
However, most of the previous interventions were performed among physicians, nurses, or other clinical staff, and very few were conducted with undergraduates [19]. Although some medical schools have included the principles of EBM as a subject within their medical curriculum, few studies have explored the barriers and enablers that students experience when studying medicine and attempting to integrate EBM into their clinical experience. The principles and application of EBM are perceived to be important for medical students, in both their current clinical training and future work as clinicians. Thus, the university restructured its curriculum and included the EBM course, which emphasized EBM practices and aimed to improve students' practical application of the tool.

EBP is the integration of the best research evidence with patients' values and clinical circumstances in clinical decision making, thus teaching EBM should be evaluated and guided by evidence of its own effectiveness [20]. More than 100 unique assessment strategies that target different objectives have been developed [20]. In this study we considered a number of instruments with reasonable validity that could evaluate some domains of EBP and target different evaluation needs. We finally chose the questionnaire developed by Johnston et al. [10]. The survey focused on the evaluation of EBP knowledge, attitude, personal application, and anticipated future use, and the questionnaire was developed and validated for the assessment of EBM education in students with similar backgrounds.

In the current study, EBM knowledge, attitudes, personal application, and anticipated future use increased in different degrees. In contrast to a previous study in which EBP-K showed the greatest change across the training [11], our study found that EBP-P had the most pronounced percentage change of scores after EBM training $(48.97 \pm 8.6 \%)$. This is because of the training's emphasis on application skills and EBP methods, and as such it is now viewed as an achievement of our EBM teaching reform. The practice of EBM depends more on

Table 2 stratified comparisons of percentage change of scores before and after an EBM training course

\begin{tabular}{lcccc}
\hline & Percentage EBP-K & Percentage EBP-A $_{\text {EB }}$ & Percentage $_{\text {EBP-P }}$ & Percentage EBP-F \\
\hline Total & $19.21 \pm 3.2 \%$ & $20.83 \pm 2.1 \%$ & $48.97 \pm 8.6 \%$ & $17.82 \pm 5.7 \%$ \\
Gender & & & & \\
$\quad$ Male & $19.23 \pm 2.2 \%$ & $20.84 \pm 2.8 \%$ & $48.96 \pm 7.9 \%$ & $17.83 \pm 1.7 \%$ \\
$\quad$ Female & $19.18 \pm 1.8 \%$ & $20.82 \pm 2.2 \%$ & $48.99 \pm 8.4 \%$ & $17.88 \pm 6.1 \%$ \\
$\quad P$ value & 0.920 & 0.200 & 0.414 & 0.925 \\
Program subtypes & & & & $17.80 \pm 5.4 \%$ \\
Five-year & $19.25 \pm 2.8 \%$ & $20.82 \pm 1.7 \%$ & $48.95 \pm 7.6 \%$ & $17.85 \pm 4.8 \%$ \\
Eight-year & $19.01 \pm 3.4 \%$ & $20.86 \pm 1.1 \%$ & $49.11 \pm 9.5 \%$ & 0.283 \\
$\quad P$ value & 0.508 & 0.094 & 0.528 & \\
\hline
\end{tabular}


application skills and methodology (in clinical studies) than on detailed knowledge. The EBP-P scores in our study are also higher than those in Cheng's study [11]. This reveals the effectiveness of practical training in EBM skills.

\section{Conclusion}

In summary, the integration of EBM in a medical curriculum improved the EBM knowledge, attitudes, personal application, and anticipated future use of such methods in a sample of 251 undergraduate medical students. A well-designed EBM training program and objective outcome instruments are necessary to ensure the effective implementation and measurement of such methods. A long-term follow-up on the effects of EBM skills on patient outcomes in future studies will further strength and validate the conclusion of our research.

\section{Additional files}

\section{Additional file 1: The finished the premedical education and clinical courses. \\ Additional file 2: Consent to participate "Evidence-Based Medicine Training" Project. \\ Additional file 3: Questionnaires of EBM skills.}

\section{Competing interests}

The authors declare that they have no conflicts of interest. The authors alone are responsible for the content and writing of the paper.

\section{Authors' contributions}

$M X, X B, L Q, Z Y$ collected the data, undertook statistical analysis and drafted the manuscript. LY, XH, MX developed the study protocol. All authors read and approved the final manuscript.

\section{Authors' information}

Xiangyu Ma, MD, PhD, is an instructor of evidence-based medicine education at the third military medical university.

Bin $\mathrm{Xu}, \mathrm{MD}, \mathrm{MPH}$, is an instructor of evidence-based medicine education at the third military medical university.

Qingyun Liu, MD, is a master student at the third military medical university. Yao Zhang, MD, PhD, is an associate professor of evidence-based medicine education.

Hongyan Xiong, MD, PhD, is a professor of evidence-based medicine education.

Yafei Li, MD, PhD, is a professor and the director of evidence-based medicine education.

\section{Acknowledgements}

This study was supported by a grant from the National Natural Science Foundation of China (grant no. 81302498) and a teaching-reforming grant from College of Preventive Medicine, Third Military Medical University in China. The authors would like to acknowledge all students who attended this study for their cooperation.

Received: 9 February 2014 Accepted: 30 June 2014 Published: 4 July 2014

\section{References}

1. Sackett DL, Rosenberg WM, Gray JA, Haynes RB, Richardson WS: Evidence based medicine: what it is and what it isn't. BMJ 1996, 312(7023):71-72.

2. Collins J: Evidence-based medicine. J Am College Radiol 2007, 4(8):551-554.
3. Kulier R, Gulmezoglu AM, Zamora J, Plana MN, Carroli G, Cecatti JG, Germar MJ, Pisake L, Mittal S, Pattinson R, Wolomby-Molondo JJ, Bergh AM, May W, Souza JP, Koppenhoefer S, Khan KS: Effectiveness of a clinically integrated e-learning course in evidence-based medicine for reproductive health training: a randomized trial. JAMA 2012, 308(21):2218-2225.

4. Finotto S, Carpanoni M, Turroni EC, Camellini R, Mecugni D: Teaching evidence-based practice: developing a curriculum model to foster evidence-based practice in undergraduate student nurses. Nurse Educ Pract 2013, 13(5):459-465.

5. Blake L, Ballance D: Teaching evidence-based practice in the hospital and the library: two different groups, one course. Med Ref Serv Q 2013, 32(1):100-110.

6. Prasad K: Teaching evidence-based medicine in resource-limited countries. JAMA 2012, 308(21):2248-2249.

7. Chakraborti C: Teaching evidence-based medicine using team-based learning in journal clubs. Med Educ 2011, 45(5):516-517.

8. Kruszewski A, Brough E, Killeen MB: Collaborative strategies for teaching evidence-based practice in accelerated second-degree programs. J Nurs Educ 2009, 48(6):340-342.

9. Johnson N, List-Ivankovic J, Eboh WO, Ireland J, Adams D, Mowatt E, Martindale S: Research and evidence based practice: using a blended approach to teaching and learning in undergraduate nurse education. Nurse Educ Pract 2010, 10(1):43-47.

10. Johnston JM, Leung GM, Fielding R, Tin KY, Ho LM: The development and validation of a knowledge, attitude and behaviour questionnaire to assess undergraduate evidence-based practice teaching and learning. Med Educ 2003, 37(11):992-1000.

11. Cheng HM, Guo FR, Hsu TF, Chuang SY, Yen HT, Lee FY, Yang YY, Chen TL Lee WS, Chuang CL, Chen CH, Ho T: Two strategies to intensify evidence-based medicine education of undergraduate students: a randomised controlled trial. Ann Acad Med Singapore 2012, 41(1):4-11.

12. Weng $Y H$, Kuo KN, Yang CY, Liao HH, Chen C, Lo HL, Lee WC, Chiu YW: Effectiveness of national evidence-based medicine competition in Taiwan. BMC Med Educ 2013, 13:66

13. Mary S, Julie J, Jennifer G: Teaching evidence based practice and research through blended learning to undergraduate midwifery students from a practice based perspective. Nurse Educ Pract 2014, 14(2):220-224.

14. Levin L, Hamama L: Teaching a systematic and evidence-based approach in an ambivalent context: a case example from Israel. Eval Program Plann 2013, 36(1):136-144.

15. Gorgon EJ, Basco MD, Manuel AT: Teaching evidence based practice in physical therapy in a developing country: a national survey of Philippine schools. BMC Med Educ 2013, 13(1):154.

16. Cooper MM: Evidence-based reform of teaching and learning. Anal Bioanal Chem 2014, 406(1):1-4.

17. Bierman KL, Derousie RM, Heinrichs B, Domitrovich CE, Greenberg MT Gill S: Sustaining high quality teaching and evidence-based curricula: follow-up assessment of teachers in the REDI project. Early Educ Dev 2013, 24(8):1194-1213.

18. Winters CA, Echeverri R: Teaching strategies to support evidence-based practice. Crit Care Nurse 2012, 32(3):49-54

19. Ilic D, Forbes K: Undergraduate medical student perceptions and use of Evidence Based Medicine: a qualitative study. BMC Med Educ 2010, 10:58.

20. Shaneyfelt T, Baum KD, Bell D, Feldstein D, Houston TK, Kaatz S, Whelan C, Green M: Instruments for evaluating education in evidence-based practice: a systematic review. JAMA 2006, 296(9):1116-1127.

doi:10.1186/1472-6920-14-133

Cite this article as: Ma et al:: Effectiveness of evidence-based medicine training for undergraduate students at a Chinese Military Medical University: a self-controlled trial. BMC Medical Education 2014 14:133. 